\title{
Evaluation of the Green's function of disordered graphene
}

\author{
W. Zhu, ${ }^{1,2}$ Q. W. Shi,,${ }^{1,2, *}$ X. R. Wang,,${ }^{2,3, \dagger}$ X. P. Wang, ${ }^{1}$ J. L. Yang, ${ }^{1}$ J. Chen, ${ }^{4,5}$ and J. G. Hou ${ }^{1}$ \\ ${ }^{1}$ Hefei National Laboratory for Physical Sciences at the Microscale, University of Science and Technology of China, \\ Hefei 230026, China \\ ${ }^{2}$ Department of Physics, The Hong Kong University of Science and Technology, Clear Water Bay, Kowloon, Hong Kong \\ ${ }^{3}$ School of Physics, Shandong University, Jinan, People's Republic of China \\ ${ }^{4}$ Electrical and Computer Engineering, University of Alberta, Alberta, Canada T6G 2V4 \\ ${ }^{5}$ National Research Council/National Institute for Nanotechnology, Alberta, Canada T6G 2M9
}

(Received 4 August 2010; revised manuscript received 22 September 2010; published 11 October 2010)

\begin{abstract}
Accurate simulations of Green's function and the self-energy function for noninteracting electrons in disordered graphenes are performed. The fundamental physical quantities such as the elastic relaxation time $\tau_{e}$, the phase velocity $v_{p}$, and the group velocity $v_{g}$ are evaluated. New features around the Dirac point are revealed, indicating that multiscattering-induced hybridization of Bloch states plays an important role in the vicinity of the Dirac point.
\end{abstract}

DOI: 10.1103/PhysRevB.82.153405

PACS number(s): 81.05.ue, 71.23.-k, 71.55.-i

\section{INTRODUCTION}

Graphene, a single layer of graphite, has been intensively studied in recent years for its many intriguing transport properties. ${ }^{1-3}$ Examples include minimum conductivity and linear carrier density dependence of conductivity. ${ }^{4,5}$ Electrons in an ideal graphene are governed by the relativistic massless Dirac equation and exhibit a linear-dispersion relation in the vicinity of the Dirac point, and zero density of state at the Dirac point. ${ }^{6}$ Among many relativistic effects, Klein paradox ${ }^{3}$ is arguably one of the most important effects that makes Dirac electrons different from the Schrödinger electrons in disordered systems. ${ }^{7}$

An in-depth understanding of Dirac electrons in disordered graphenes requires a better knowledge of the selfenergy function to extract such fundamental physical quantities as the phase velocity $v_{p}$, the group velocity $v_{g}$, and the elastic relaxation time $\tau_{e}$. However, it is known ${ }^{8}$ that the accurate and reliable calculation is quite difficult and nontrivial, although various approximations have been employed in different theoretical studies. So far, almost all calculations $^{9,10}$ on electron properties in disordered graphenes were performed without fully considering the disorder effects. The wave nature of Dirac electrons is more pronounced near the Dirac point because of very large electron wavelength there. It is known that interference of multiscattering leads to the weak localization and the Anderson localization in conventional disordered electron systems. ${ }^{11}$ Quantum interference also plays important roles in coherent wave propagation through quasirandom ${ }^{12}$ and random media. ${ }^{13,14}$ As it will be shown below, hybridization of Bloch states near the Dirac point in realistic disordered graphenes is essential in understanding the diffusion properties although previous calculations ${ }^{9,10}$ can capture the essential physics far away from the Dirac point.

In this Brief Report, we present a systematic method for the precise computation of Green's function and the selfenergy of large size disordered graphenes. We extract accurate $\tau_{e}, v_{p}$, and $v_{g}$ values from the spectral function $A(\mathbf{k}, E)$ derived from the self-energy function. Compared the results from the self-consistent Born approximation (SCBA), it is found that the $\tau_{e}$ is overestimated by the SCBA in the strong disorder regime. We show that both $v_{p}$ and $v_{g}$ deviate significantly from their unrenormalized values and exhibit substantial energy dependence, especially near the Dirac point. The effective group velocity is larger than the effective phase velocity but substantially lower than its unrenormalized value when the mixing of the different Bloch states is dominant. Moreover, we generalize the Einstein relation to calculate the conductivity of the disordered graphene.

\section{MODEL AND METHOD}

$\pi$ electrons of undoped graphene can be modeled by a tight-binding Hamiltonian on a honeycomb lattice of two sites per unit cell, $H_{0}=t \sum_{\langle i j\rangle}|i\rangle\langle j|+$ H.c., where $t=-2.7 \mathrm{eV}$ is the hopping energy. The corresponding eigenvalues and eigenstates of $H_{0}$ near the Dirac point are $E_{k, \pm}= \pm \hbar v_{F}^{0} k$ and $|\mathbf{k} \pm\rangle=\left(|\mathbf{k} A\rangle \pm e^{i \phi(\mathbf{k})}|\mathbf{k} B\rangle\right) / 2$, respectively. ${ }^{4,15}$ Here, $v_{F}^{0}$ is the unrenormalized Fermi velocity and $\hbar$ is the Planck's constant. $A$ and $B$ stand for $A$ and $B$ sublattices. $\phi(\mathbf{k})$ is the polar angle of the momentum $\mathbf{k}$ and $|\mathbf{k} A(B)\rangle$ $=\frac{1}{\sqrt{N_{A(B)}}} \sum_{r_{A(B)}} e^{i \mathbf{k} \cdot \mathbf{r}_{A(B)}}\left|r_{A(B)}\right\rangle$, where $\mathbf{r}_{A(B)}$ is the position vector of $A(B)$ lattice and $N_{A(B)}$ is the total $A(B)$-lattice points. The plus (minus) sign denotes the conduction (valence) band. The Green's function of the clean graphene is, in a diagonal basis, $\quad G_{0}(\mathbf{k}, E)=\frac{1}{E+i 0^{+}-\hbar v_{F}^{0} k}|\mathbf{k}+\rangle\left\langle\mathbf{k}+\left|+\frac{1}{E+i 0^{+}+\hbar v_{F}^{0} k}\right| \mathbf{k}-\right\rangle\langle\mathbf{k}-| . \quad \mathrm{A}$ weak pointlike disorder is introduced through $V=\sum \epsilon_{i}|i\rangle\langle i|$ with $n_{\text {imp }}$ randomly distributed impurity sites where the onsite energy $\epsilon_{i}$ of each impurity can take $-V_{0}$ or $V_{0}$ (measured in the unit of $t$ ) with equal probability. In this Brief Report, $V_{0}$ is chosen $V_{0}<3$ to guarantee no resonant states around the Dirac point. ${ }^{16,17}$ A dimensionless parameter $\alpha=\frac{n_{i m p} V_{0}^{2} A_{c}}{2 \pi\left(\hbar v_{F}^{0}\right)^{2}}$ can be used to characterize disorder strength. Here, $A_{c}$ is the area of the unit cell. Our calculation shows that physical quantities such as self-energy are only determined by the parameter $\alpha{ }^{18}$

The ensemble-averaged Green's function is defined as $G(\mathbf{k} \pm, E)=\left\langle\mathbf{k} \pm\left|\frac{1}{E+i \eta-H_{0}-V}\right| \mathbf{k} \pm\right\rangle$, where the bar means the en- 

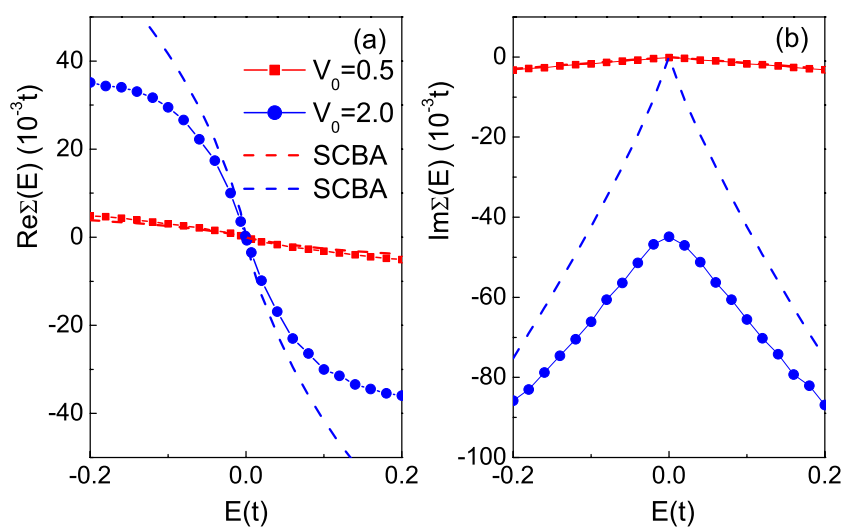

FIG. 1. (Color online) (a) Real part of self-energy as a function of energy. (b) Imaginary part of self-energy as a function of energy. The squares and circles are our numerical calculations for the disorder concentration $n_{\text {imp }} / N=10 \%$ and $V_{0}=0.5$ and $V_{0}=2.0$, respectively. Dashed lines are for the SCBA results for the same disorder. Energy is measured in the units of $t$.

semble average. It can be calculated by using the welldeveloped Lanczos recursive method. ${ }^{16,19,20}$ In order to obtain an accurate ensemble-averaged Green's function near the Dirac point with high-energy resolution, ${ }^{21}$ a large sample containing $N=L_{x} \times L_{y} \simeq 6.0$ millions carbon atoms (2400 $\times 2400)$ and over 1000 ensembles are used in our simulation, where $L_{x}$ and $L_{y}$ is the number of atoms in $x$ and $y$ directions, respectively. The large lattice samples guarantee that the calculated Green's function is free of finite-size errors. ${ }^{22}$ The periodic boundary condition has been used and the wave vectors satisfy $k_{x}=n_{x} 4 \pi / 3 a L_{x}$ and $k_{y}=n_{y} 4 \pi / \sqrt{3} a L_{y}$, where $n_{x(y)}$ is an integer and $a$ is the lattice constant.

\section{SELF-ENERGY FUNCTION}

Figure 1 shows the calculated (a) real and (b) imaginary parts of the self-energy function for $n_{i m p} / N=10 \%$ and $V_{0}$ $=0.5$ (squares) and 2.0 (circles), respectively. The self-energy function is defined based on the Dyson' equation $G(\mathbf{k}, E)$ $=G_{0}(\mathbf{k}, E)+G_{0}(\mathbf{k}, E) \Sigma(\mathbf{k}, E) G(\mathbf{k}, E), \quad$ thus $\quad \Sigma(\mathbf{k}, E)$ $=G_{0}^{-1}(\mathbf{k}, E)-G^{-1}(\mathbf{k}, E){ }^{8}$ In principle, the self-energy function depends on energy $E$ and wave vector $\mathbf{k}$. However, our simulation shows that the self-energy function is not sensitive to wave vector $\mathbf{k}$ while the one-particle Green's function depends on both $E$ and $\mathbf{k}$. This is not surprising since the scatterer size is much smaller than the electron wavelength near the Dirac point so that the inhomogeneous structure of disordered graphene can be well described by an effective homogeneous medium. This is also why the self-energy function is assumed to be $\mathbf{k}$ independent in many perturbative calculations. Our finding validates these assumptions. ${ }^{23}$

Our calculation should be compared with widely used results from the SCBA that predicts ${ }^{15,24}$

$$
\Sigma(E)= \begin{cases}-E / 2 \alpha-i \Gamma_{0}, & |E| \ll \Gamma_{0}, \\ -2 \alpha(E+i \pi \alpha|E|) \ln \left|\frac{E_{c}}{E}\right|-i \pi \alpha|E|, & |E| \gg \Gamma_{0},\end{cases}
$$

where $\Gamma_{0}=E_{c} e^{-1 / 2 \alpha}$ ( $E_{c}$ is the cut-off energy). As shown in Fig. 1, SCBA results agree well with our exact self-energy

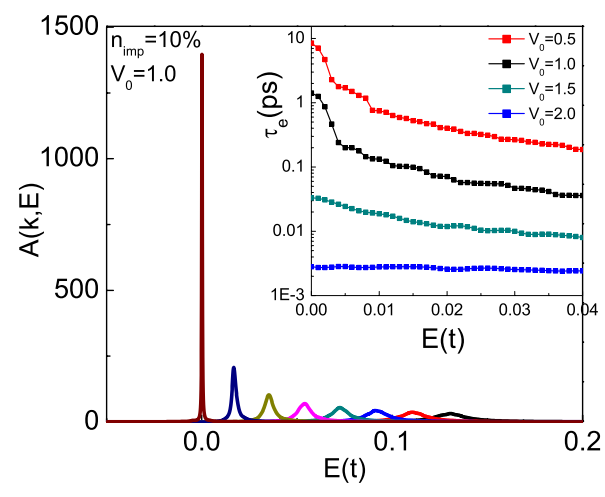

FIG. 2. (Color online) Single-particle spectral function $A(\vec{k}$ ,$+ E$ ) plotted as a function of energy $E$ at several $k$ points (from left to right) $k=0.000,0.014,0.028,0.042,0.056,0.070,0.084$, and 0.098 (or $n_{x}=0-56$ ) along the $k_{x}$ direction. Model parameters are $n_{\text {imp }} / N=10 \%$ and $V_{0}=1.0$. Inset: the energy dependence of singleparticle relaxation time $\tau_{e}$ for $V_{0}=0.5-2.0$ and $n_{i m p} / N=10 \%$.

function for very weak disorder $\left(V_{0}=0.5\right.$ and $\left.n_{\text {imp }} / N=10 \%\right){ }^{25}$ When the disorder strength increases several times $\left(V_{0}=2.0\right.$ or $\left.\alpha \sim 0.07\right)$, the perturbative results cannot capture the main features, especially near the Dirac point. The discrepancy is obvious for $\operatorname{Im} \Sigma(0)$ as shown in Fig. 1(b). Im $\Sigma(E)$ at the Dirac point is $\Gamma_{0} \sim 10^{-3} t\left[E_{c}=\sqrt{3} t\right.$ (Ref. 6)] from the SCBA while our exact value is $\operatorname{Im} \Sigma(0)$ $\geq 10^{-2} t$. Therefore, the true broadening of states at or near the Dirac point is much bigger than what is predicted by SCBA. This difference can be attributed to the mixture of Bloch states caused by the impurities. ${ }^{5,26}$ Furthermore, the level-repulsion effect pushes all energy level toward the Dirac point so that the density of states at the Dirac point increases more in the presence of impurities (see inset of Fig. 4 below). Therefore, the impurities increase the imaginary part of the self-energy function (directly associated with the density of states) at the Dirac point. When the wavelength becomes short, and the quantum interference as well as the Bloch state mixing are less important, $\operatorname{Im} \Sigma(E)$ are determined by the disorder scattering. The difference between our exact simulation and that of the SCBA is small, as shown in Fig. 1(b).

\section{SPECTRAL FUNCTION}

The single-particle spectral function relates to the Green's function through $A(\mathbf{k} \pm, E)=-\operatorname{Im} G(\mathbf{k} \pm, E) / \pi .^{23}$ Figure 2(a) is $A(\mathbf{k}+, E)$ for $k_{y}=0, V_{0}=1, n_{\text {imp }} / N=10 \%$, and various $k_{x}$ (in unit of $a^{-1}$ ) (curves from the left to the right in the figure) ranging from $0.0\left(n_{x}=0\right)$ to $0.098\left(n_{x}=56\right)$. In the absence of disorders, the spectral function $A_{0}(\mathbf{k} \pm, E)$ is a delta function, reflecting that the wave vector $\mathbf{k}$ is a good quantum number and has all its spectral weight precisely at the energy $E=E_{k \pm}$. In the presence of disorders, the translational symmetry is broken and the spectral function is broadened, resulting from the disorder scattering effect. The widths of the spectral function is given by $\operatorname{Im} \Sigma(E)$ that measures the elastic relaxation lifetime $\tau_{e}, \tau_{e}=\frac{\hbar}{-2 \operatorname{Im} \Sigma(E)}$. Therefore, the elastic scattering relaxation time is then akin to 

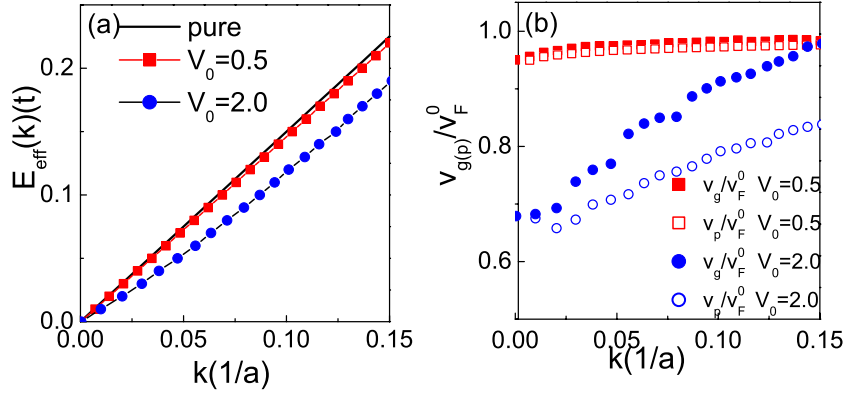

FIG. 3. (Color online) (a) Effective energy dispersion relation for ideal graphene and disordered graphenes with $n_{\text {imp }} / N=10 \%$ and $V=0.5$ (filled squares) and $V=2.0$ (filled circles). (b) The ratio $v_{g} / v_{F}^{0}$ (filled symbols) and $v_{p} / v_{F}^{0}$ (open symbols) as a function of wave number $k$ (in units of $1 / a$ ).

$\operatorname{Im} \Sigma(E)$, and $\tau_{e}$ around the Dirac point is mainly determined by the Bloch state mixing and level repulsion effect. ${ }^{27}$ Far away from the Dirac point, the lifetime is mainly attributed to the disorder scattering. As shown in the inset of Fig. 2, lifetime become shorter as the wave vector increases. Physically, this is because the density of states $\rho$ increases linearly with energy and the disorder scattering effects become larger, in qualitative agreement with the prediction of SCBA. ${ }^{15,24,28}$

\section{EFFECTIVE BAND VELOCITY}

Dirac electron propagation velocities including the group velocity $v_{g}$ and phase velocity $v_{p}$ are also greatly modified by disorder effects. These quantities relate to the shape of the dispersion relation that is the roots of $E-E_{0}(k)-\operatorname{Re} \Sigma(E)$ $=0 .{ }^{29,30}$ One can also extract the dispersion relation from the peak of the spectral function $A(\mathbf{k}, E)$ for a given $\mathbf{k}$. Figure $3\left(\right.$ a) is our exact dissipation curve $E_{\text {eff }}(k)$ that is linear for very weak disorder $(\alpha<0.01)$. However, when the disorder strength becomes large enough $(\alpha \sim 0.1)$, the $E_{\text {eff }}(k)$ is concave near the Dirac point, indicating the reduction in both the group and phase velocities.

Figure 3(b) is the $k$ dependence of the group and phase velocities that can be obtained from $v_{g}=\partial E_{\text {eff }}(k) / \partial k$ and $v_{p}$ $=E_{\text {eff }} / k$. For very weak disorder $\alpha=0.01, v_{g}$ and $v_{p}$ are not too much different from the unrenormalized velocity $v_{F}^{0}$ with only 5\% reduction at the Dirac point as shown in the figure. When the disorder strength increase several times $\alpha=0.07$, the renormalized $v_{g}$ is higher than $v_{p}$, and both $v_{g}$ and $v_{p}$ are reduced by a large percentage near the Dirac point. It shows that disorder not only renormalizes the Dirac electron velocities but also changes the linear-dispersion relation. ${ }^{26}$ The observation that significant reduction in velocities occur at the Dirac point reflects that the Dirac electrons near the Dirac point are more sensitive to the disorder.

One can use the renormalization factor $Z$ defined as $Z$ $=\left[1-\frac{\partial \operatorname{Re} \Sigma(E, \mathbf{k})}{\partial E}\right]^{-1}$ to measure the disorder effect on electronic structure. ${ }^{31}$ Its value equals the ratio $v_{g} / v_{F}^{0}$. As shown in Fig. 3(b), $Z$ is very close to 1.0 for very weak disorder. In this regime, the Bloch state is still a good starting point for understanding disordered graphene and the transport properties are expected to be described by the quasiclassical Boltz-

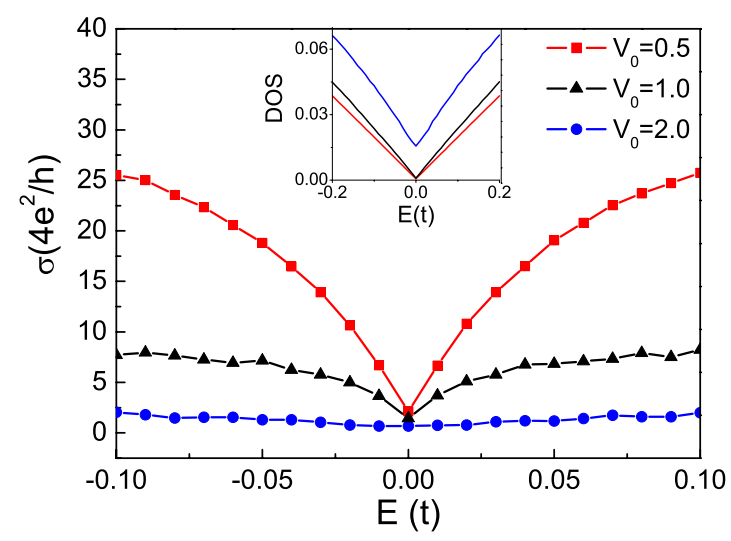

FIG. 4. (Color online) Conductivity as a function of charge density. The model parameters are $n_{\text {imp }} / N=10 \%$ and $V_{0}=0.5,1.0$, and 2.0. Inset: density of states for the same parameters.

mann theory. When the disorder strength increases several times, $Z$ is much smaller than 1.0 around the Dirac point. This unusual feature directly reflects that a Bloch state around the Dirac point strongly couples with other nearby Bloch states. These hybridized waves can also be expanded in a complete set of Bloch states $\left[\phi_{\mathbf{k}}(r)=e^{i \mathbf{k} \cdot \mathbf{r}} u(r)\right]: \Psi(r)$ $=\Sigma_{\mathbf{k}} c_{\mathbf{k}} \phi_{\mathbf{k}}(r)$, where $c_{\mathbf{k}}=\left\langle\phi_{\mathbf{k}} \mid \psi\right\rangle$. If $Z$ approaches 1.0, the system is dominated by a single "majority representation" wave vector $\mathbf{k}_{0}$ while $\Psi(r)$ essentially behaves as a Bloch periodic state $\phi_{\mathbf{k}_{0}}(r)$. However, $\mathbf{k}_{0}$ is not a good approximate quantum number anymore when $Z$ is much smaller than 1.0. Therefore, Bloch states would not be a good starting point to perform perturbative calculations. In fact, similar features had been observed in semiconductor alloys and been termed nonBloch nature of alloy states. ${ }^{32}$ This is also why the SCBA method cannot produce accurate enough results. ${ }^{26}$ Furthermore, this nontrivial feature could lead to intriguing transport and optical properties. For example, linear-dispersion relation leads to $E_{n}= \pm v_{F}^{0} \sqrt{2 e \hbar n B}(n=0,1,2 \ldots)$ Landau-level (LL) series. Since the LLs come from the quenching of electrons' kinetic energies, lower group velocity near the Dirac point means the energy gap between $n=0$ LL and $n$ $= \pm 1$ LL should be substantially reduced by the disorders while that between $n= \pm 1$ LLs and $n= \pm 2$ LLs will be affected less by disorder. This is indeed what was observed in a recent experiment of cyclotron resonance. ${ }^{33}$

\section{CONDUCTIVITY}

Assuming that the Einstein relation for the Dirac electrons is valid, one can obtain the conductivity from $\sigma=e^{2} D \rho$, where $e$ is the electron charge and $D=v_{g}^{2} \tau_{t r} / 2$ is the diffusion coefficient, and $\tau_{t r}$ denotes the transport relaxation time and satisfies the relationship $\tau_{t r}=2 \tau_{e}$ for the pointlike disorder. ${ }^{5}$ Figure 4 is the conductivity calculated based on the transport relaxation time $\tau_{t r}=2 \tau_{e}$ in Fig. 2 . The group velocity $v_{g}$ is shown in Fig. 3. The density of states is obtained by $\rho(E)=$ $-\frac{A_{c}}{\pi} \int_{0}^{k_{c}} \frac{d \vec{k}}{(2 \pi)^{2}} A(\vec{k}, E)$ as shown in the inset of Fig. 4. For very weak disorder, the conductivity is sublinear in the Fermi energy. While increasing the disorder strength, the conductivity depends weakly on the Fermi energy or the carrier density, 
which agrees with the prediction of Boltzmann theory for short-range scatterers. ${ }^{15}$ At the Dirac point, when combining the self-energy function with the Kubo formula, the conductivity is predicted to be the universal value $\sigma_{x x}(0)=4 e^{2} / \pi h .^{15}$ However, our calculations find that $\sigma_{x x}(0)$ takes the values $8.2 e^{2} / h, 5.9 e^{2} / h$, and $2.7 e^{2} / h$ for $\alpha=0.0046,0.018$, and 0.07 , respectively and shows nonuniversal behavior.

\section{SUMMARY}

In conclusion, we studied pointlike disorder effects on the one-electron properties of graphene. The exact ensembleaveraged Green's function is obtained from a large-scale real-space calculation. Through the analysis of self-energy and spectral functions, we conclude that the single-particle lifetime reduction and the linear-dispersion relation are modified by the hybridization of the Bloch states. The strong hybridization of Bloch states near the Dirac point indicates the Bloch-Boltzmann theory need to be modified in disordered graphene. Our approach is very general and robust, and is applicable to other disordered systems.

\section{ACKNOWLEDGMENTS}

This work is partially supported by NNSF of China (Grants No. 10974187, No. 10874165, and No. 50721091), by NKBRP of China under Grant No. 2006CB922000, and by KIP of the Chinese Academy of Sciences (Grant No. KJCX2-YW-W22). J.C. acknowledges support from the NRC/NINT and NSERC (Grant No. 245680). X.R.W. acknowledges the support of Hong Kong RGC grants (Grants No. 604109, No. RPC10SC05, and No. HKU10/CRF/08HKUST17/CRF/08). *phsqw@ustc.edu.cn

†phxwan@ust.hk

${ }^{1}$ K. S. Novoselov et al., Science 306, 666 (2004).

${ }^{2}$ Y. Zhang et al., Nature (London) 438, 201 (2005).

${ }^{3}$ A. K. Geim et al., Nature Mater. 6, 183 (2007).

${ }^{4}$ A. H. Castro Neto et al., Rev. Mod. Phys. 81, 109 (2009).

${ }^{5}$ S. Das Sarma, S. Adam, E. Hwang, and E. Rossi, arXiv:1003.4731 (unpublished).

${ }^{6}$ Z. F. Wang, R. Xiang, Q. W. Shi, J. Yang, X. Wang, J. G. Hou, and J. Chen, Phys. Rev. B 74, 125417 (2006).

${ }^{7}$ Y. Y. Zhang, J. Hu, B. A. Bernevig, X. R. Wang, X. C. Xie, and W. M. Liu, Phys. Rev. Lett. 102, 106401 (2009); Y. Zhang, J. P. Hu, B. A. Bernevig, X. R. Wang, X. C. Xie, and W. M. Liu, Phys. Rev. B 78, 155413 (2008).

${ }^{8}$ E. N. Economou, Green's Functions in Quantum Physics (Springer, New York, 2006).

${ }^{9}$ E. H. Hwang and S. Das Sarma, Phys. Rev. B 77, 195412 (2008).

${ }^{10}$ M. I. Katsnelson et al., Philos. Trans. R. Soc. London, Ser. A 366, 195 (2008).

${ }^{11}$ P. A. Lee et al., Rev. Mod. Phys. 57, 287 (1985).

${ }^{12}$ X. R. Wang, Phys. Rev. B 51, 9310 (1995); 53, 12035 (1996).

${ }^{13}$ M. C. W. van Rossum et al., Rev. Mod. Phys. 71, 313 (1999).

${ }^{14}$ M. P. van Albada, B. A. van Tiggelen, A. Lagendijk, and A. Tip, Phys. Rev. Lett. 66, 3132 (1991).

${ }^{15}$ T. Ando, J. Phys. Soc. Jpn. 75, 074716 (2006); N. H. Shon and T. Ando, ibid. 67, 2421 (1998).

${ }^{16}$ S. Wu, L. Jing, Q. Li, Q. W. Shi, J. Chen, H. Su, X. Wang, and J. Yang, Phys. Rev. B 77, 195411 (2008); V. M. Pereira, F. Guinea, J. M. B. Lopes dos Santos, N. M. R. Peres, and A. H. Castro Neto, Phys. Rev. Lett. 96, 036801 (2006); B. L. Huang, et al., EPL 88, 68005 (2009); and N. M. R. Peres, F. Guinea, and A. H. Castro Neto, Phys. Rev. B 73, 125411 (2006).

${ }^{17}$ Resonant states will lead to some new physical features, which needs further study.

${ }^{18}$ Other uncorrelated short-range impurity potential (such as Anderson disorder) produces similar results.

${ }^{19}$ J. K. Cullum and R. A. Willoughby, Lanczos Algorithms for Large Symmetric Eigenvalue Problem (Birkhäuser, Boston, 1985).
${ }^{20}$ E. Dagotto, Rev. Mod. Phys. 66, 763 (1994); L. C. Davis, Phys. Rev. B 28, 6961 (1983); B. Bäuml, G. Wellein, and H. Fehske, ibid. 58, 3663 (1998); S. M. Anlage and D. L. Smith, ibid. 34, 2336 (1986).

${ }^{21}$ A small artificial cut-off energy $\eta$ is used to simulate the infinitesimal imaginary energy in Green's function. We chose $\eta$ $\sim 0.01 \mathrm{meV}$ in this Brief Report, which is much less than the energy resolution in recent angle-resolved photoemission spectroscopy experiments (Ref. 34).

${ }^{22}$ It has been checked that the averaged Green's functions do not change when the sample size is doubled to $4800 \times 4800$ or reduced to $1200 \times 1200$.

${ }^{23}$ A. Altland and B. Simons, Condensed Matter Field Theory (Cambridge University Press, Cambridge, 2006).

${ }^{24}$ P. M. Ostrovsky, I. V. Gornyi, and A. D. Mirlin, Phys. Rev. B 74, 235443 (2006).

${ }^{25}$ T. Fukuzawa et al., J. Phys. Soc. Jpn. 78, 094714 (2009).

${ }^{26}$ I. L. Aleiner and K. B. Efetov, Phys. Rev. Lett. 97, 236801 (2006); it is found that SCBA neglects most terms of equal order in perturbation theory.

${ }^{27}$ M. Calandra and F. Mauri, Phys. Rev. B 76, 205411 (2007).

${ }^{28}$ K. Nomura and A. H. MacDonald, Phys. Rev. Lett. 96, 256602 (2006); 98, 076602 (2007).

${ }^{29}$ G. D. Mahan, Many-Particle Physics, 3rd ed. (Kluwer Academic, New York, 2000).

${ }^{30}$ K. Tarafder, A. Chakrabarti, K. K. Saha, and A. Mookerjee, Phys. Rev. B 74, 144204 (2006).

${ }^{31}$ C. H. Park, F. Giustino, M. L. Cohen, and S. G. Louie, Phys. Rev. Lett. 99, 086804 (2007).

${ }^{32}$ L.-W. Wang, L. Bellaiche, S.-H. Wei, and A. Zunger, Phys. Rev. Lett. 80, 4725 (1998); Y. Zhang, A. Mascarenhas, and L.-W. Wang, ibid. 101, 036403 (2008).

${ }^{33}$ E. A. Henriksen, P. Cadden-Zimansky, Z. Jiang, Z. Q. Li, L.-C. Tung, M. E. Schwartz, M. Takita, Y.-J. Wang, P. Kim, and H. L. Stormer, Phys. Rev. Lett. 104, 067404 (2010); for details please see our interpretation to this cyclotron resonance experiment (Ref. 35).

${ }^{34}$ A. Damascelli et al., Rev. Mod. Phys. 75, 473 (2003).

${ }^{35}$ W. Zhu, Q. W. Shi, J. G. Hou, and X. R. Wang, Phys. Rev. Lett. 105, 159703 (2010). 